\title{
Electromagnetic Imaging of Two-Dimensional Geometries by Multipulse Interference Using the Inverse FDTD Method
}

\author{
Naoki Okada and James B. Cole \\ Graduate School of Systems and Information Engineering, University of Tsukuba, 1-1-1 Tennodai, Tsukuba, Ibaraki 305-8573, Japan \\ Correspondence should be addressed to Naoki Okada; okada@cavelab.cs.tsukuba.ac.jp
}

Received 24 January 2014; Revised 10 March 2014; Accepted 10 March 2014; Published 31 March 2014

Academic Editor: Joseph Rosen

Copyright ( 2014 N. Okada and J. B. Cole. This is an open access article distributed under the Creative Commons Attribution License, which permits unrestricted use, distribution, and reproduction in any medium, provided the original work is properly cited.

\begin{abstract}
The size, shape, and location of unknown objects in the ground and in the body can be estimated by an electromagnetic imaging technique. An imaging approach to clear detection of two-dimensional geometries is proposed in this paper. Based on the inverse finite-difference time-domain (FDTD) method, a phase interference technique using multidirectional pulses is employed. The advantage of the proposed method is that it can clearly reconstruct the geometry in a simple calculation. Sample imaging results are demonstrated. The analysis of the FDTD results shows that the detectable object size is limited by the incident wavelength and the measurement spacing and illustrates the detectability of multiple objects.
\end{abstract}

\section{Introduction}

Electromagnetic imaging is an inverse scattering technique to estimate unknown objects. The scattered wave from an object carries information about its geometry, size, and location. The original object shape can be reconstructed by numerically time reversing the scattering process. The inverse scattering technique has been widely studied for various applications [15] such as computed tomography, nondestructive evaluation, and geophysical remote sensing.

A variety of electromagnetic imaging techniques [6-9] have been proposed based on the finite-difference timedomain (FDTD) method. FDTD works in the time domain with arbitrary structures $[10,11]$ and thus is suitable to calculate inverse scattering problems.

One of the problems of electromagnetic imaging is to reconstruct the detailed geometry of original objects. Previous works successfully detected the position and size of the two- and three-dimensional objects [5-9], but the reconstructed geometries were unclear even for an object immersed in a uniform medium [12-18]. A comparatively successful imaging of the detailed geometry was demonstrated using phase interference with inverse FDTD simulations, in which the scattered field was calculated, and then using time reversal; the original shape was extracted from the interference patterns [19]. However this requires both the scattered electric and magnetic fields in the entire space, and it is difficult to clearly discern the original boundaries due to the continuities of electromagnetic fields unless the original shape is used in the visualization.

In this paper, we propose a multipulse interference technique using the inverse FDTD method to improve the detection of geometrical details. In Section 2, we introduce the fundamentals of the proposed approach and demonstrate the imaging for some basic geometries. In Section 3, the sensitivity of our method to small and multiple objects is verified. In Section 4, the results are summarized.

\section{Multipulse Interference Technique}

In Section 2.1, the FDTD configuration for the electromagnetic imaging is defined. In Section 2.2, the imaging procedure of our multipulse interference technique is described. In Section 2.3, an example imaging for a square is demonstrated and our imaging quality is compared with the conventional interference technique. In Section 2.4, numerical results for some geometries, refractive indices, and polarizations are verified. 


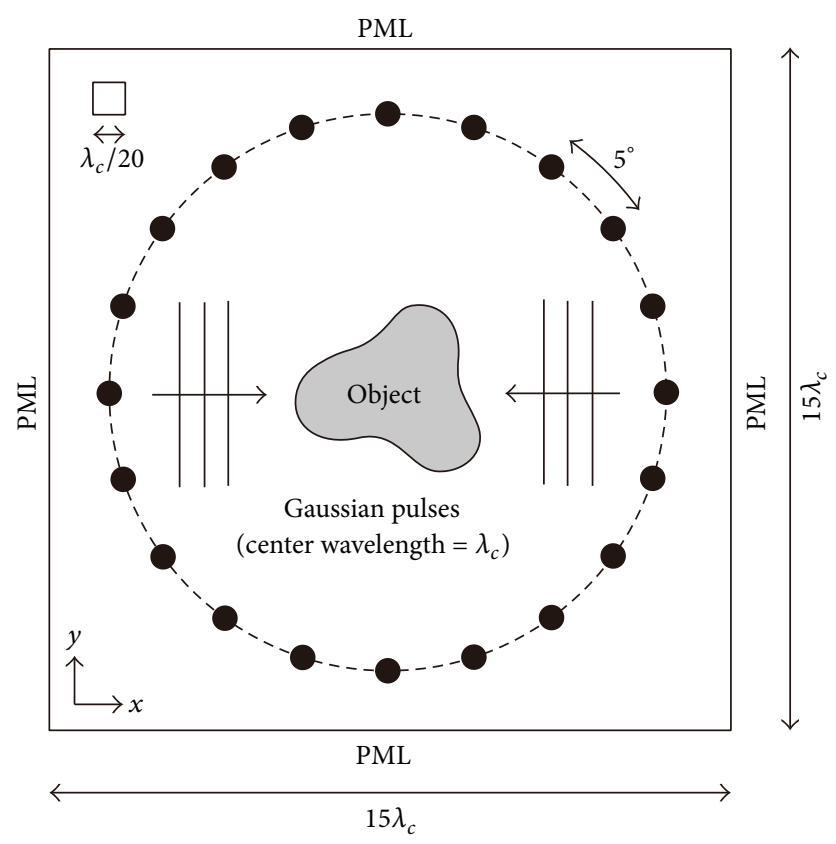

FIGURE 1: FDTD configuration. A pair of Gaussian pulses of scalable center wavelength $\lambda_{c}$ propagates along $\pm x$-axis in the TE polarization and impinges on the object. Dots spaced at $5^{\circ}$ intervals on the circumference of a circle of radius $6 \lambda_{c}$ are measurement points. Grid spacing and computational domain terminated by PML absorbing boundaries are depicted.

2.1. FDTD Configuration. The conventional phase interference technique [19] must detect both the scattered electric and magnetic fields in the entire space (all grid points), but this is the impractical measurement actually. To more closely mimic what might be experimentally observed, we employ a circular array of measurement points in common use [5, 15-18]. Figure 1 shows our FDTD configuration. In order to illuminate the object over a wide range, a pair of opposing Gaussian pulses propagating along the $\pm x$-axis is employed (the plane wave is not suitable because it makes the temporal discontinuity when the detection is terminated). The center wavelength is $\lambda_{c}$ and the beam width of the pulse is $3 \lambda_{c}$, where $\lambda_{c}$ is the arbitrary constant for scalability. The polarization is the transverse electric (TE) mode, in which the electromagnetic fields reduce to three nonzero components $H_{x}, H_{y}$, and $E_{z}$. Scattered fields are observed at measurement points spaced at $5^{\circ}$ intervals on the circumference of a circle of radius $6 \lambda_{c}$. In the FDTD calculation, the grid spacing is $\lambda_{c} / 20$ according to the accuracy considerations given in [20]. The computational domain of $15 \lambda_{c} \times 15 \lambda_{c}$ is terminated by perfectly matched layer (PML) absorbing boundaries [11].

2.2. Imaging Procedure. Our electromagnetic imaging is performed using the following steps: (1) observation, (2) imaging, and (3) combination.

(1) The forward propagating scattered wave for the pulse pair is calculated using the FDTD method. The scattered electric fields are observed on the measurement points for 20 wave periods (enough time to detect primary scattered waves) after introducing the initial pulses.

(2) The object is removed from the computational domain in Figure 1, and the scattered fields sampled at the measurement points are time-reversely input as point sources (on the measurement points). Visualizing the intensity distribution, the inverse propagation is calculated using FDTD until the primary scattered pulse pair impinges on each other. The original shape emerges in the interference pattern of intensity, because the equiphase plane caused by reflection delay is parallel to the object surface.

(3) Iterating steps 1 and 2 with a pulse pair incident along a different direction, the reconstructed images are obtained. To get a clear image independent of pulse direction, the separate intensity distributions are summed.

The crucial difference between the proposed method and the conventional interference technique [19] is the use of the pulse pair. The incident pulse should illuminate the object surface to detect the reflected wave from the surface, and thus the single directional pulse in the conventional method is not enough to accurately reconstruct the shape. The multipulse interference improves the reproducibility of original boundaries dramatically as discussed in Section 2.3. Additionally, our approach without iterative processes is performed in a much simpler calculation than statistical optimization [21].

2.3. Example Imaging for a Square. We illustrate the details of our approach by applying it to a square object. Figure 2 shows the imaging results for a square of side $3 \lambda_{c}$. The refractive indices inside and outside the object are 3.5 (silicon in microwave region) and 1 (air), respectively. Figure 2(a) depicts the original shape (scale bar is $3 \lambda_{c}$ ). A pair of opposing pulses is input along the $\pm x$-axis and the scattered $E_{z}$ fields are detected on the probe array. Using the sampled scattered fields, time-reversed propagation is carried out. Figure 2(b) shows the intensity distribution of the scattered $E_{z}$ field due to the interference of the primary scattered pulses. The original shape is successfully reconstructed in the interference patterns, but the boundaries parallel to the $x$ axis are obscure compared with those parallel to the $y$-axis. This is because the incident pulse pair propagating along the $\pm x$-axis mainly interacts with the boundaries parallel to the $y$-axis. Hence using a pulse pair propagating along the $\pm y$ axis clarifies the boundaries parallel to the $x$-axis as shown in Figure 2(c). In order to obtain a clear image independent of pulse direction, we use pulse pairs propagating along the $x$ - and $y$-axes. However, the interference of electromagnetic waves propagating perpendicularly to each other gives rise to diagonal fringe patterns, and such intermittent boundaries are obscure. To circumvent this problem, we add the two separately calculated intensity distributions shown in Figures 2(b) and 2(c). Figure 2(d) shows the combined image (sum of the two intensity distributions). All boundary shapes 


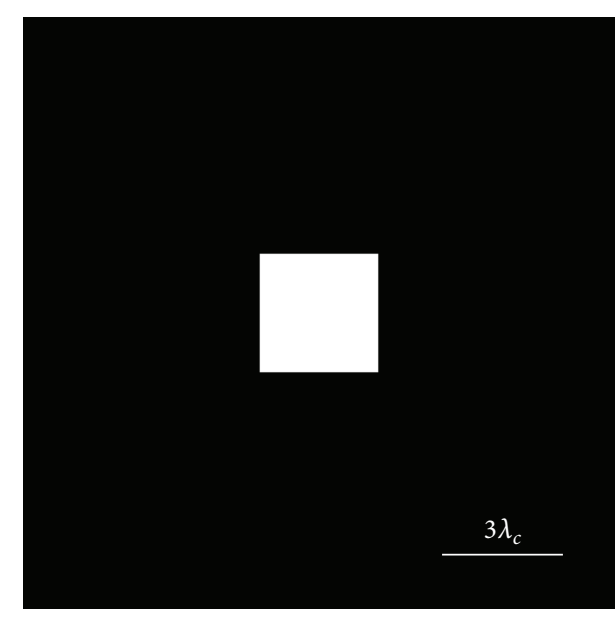

(a)

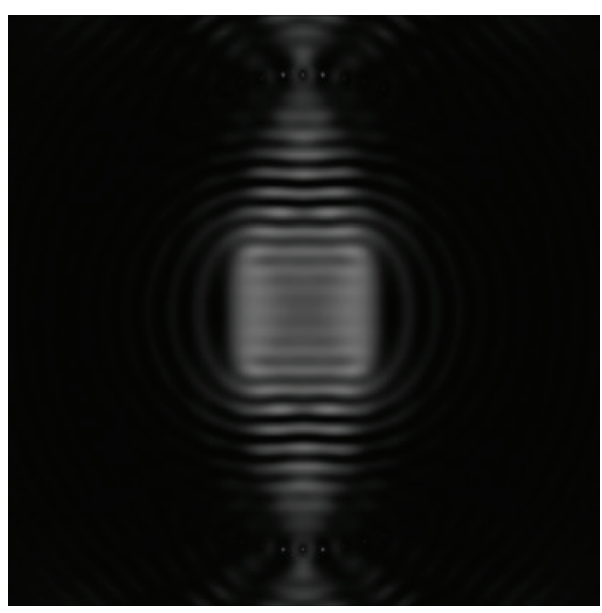

(c)

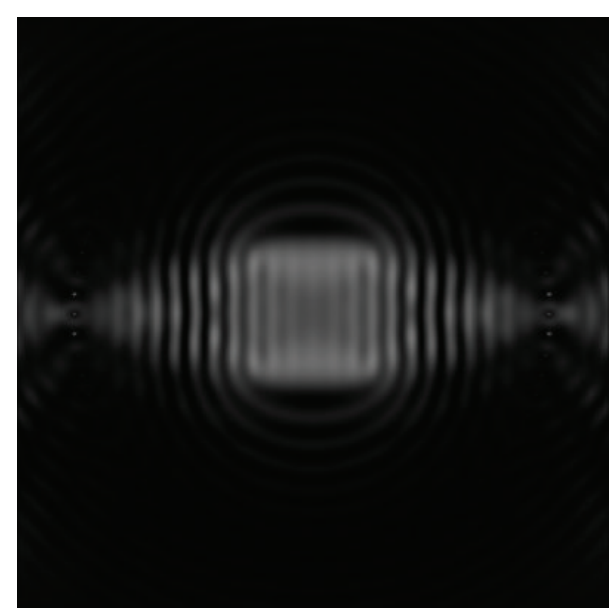

(b)

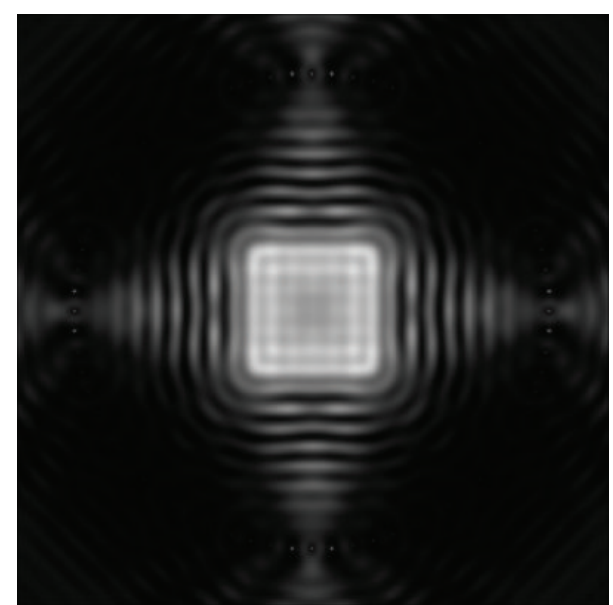

(d)

FIGURE 2: Electromagnetic imaging for a square of side $3 \lambda_{c}$. (a) Original shape (scale bar is $3 \lambda_{c}$ ). (b) Reconstructed intensity due to a pulse pair on $x$-axis. (c) Reconstructed intensity due to a pulse pair on $y$-axis. (d) Sum of intensity distributions of (b) and (c) yields an image reconstruction.

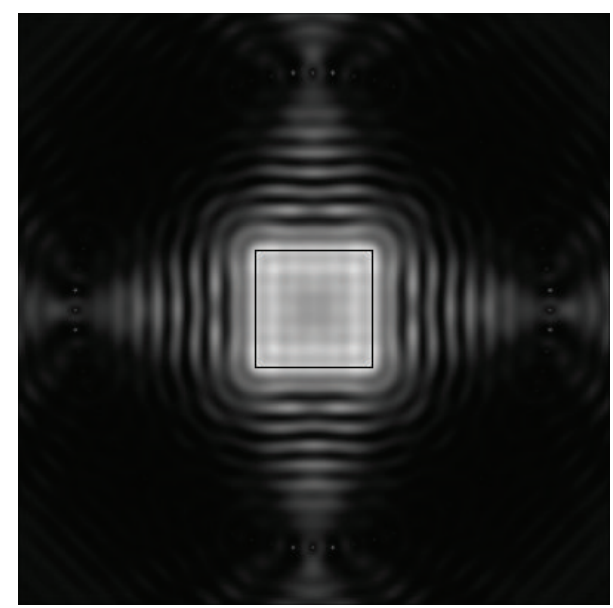

(a)

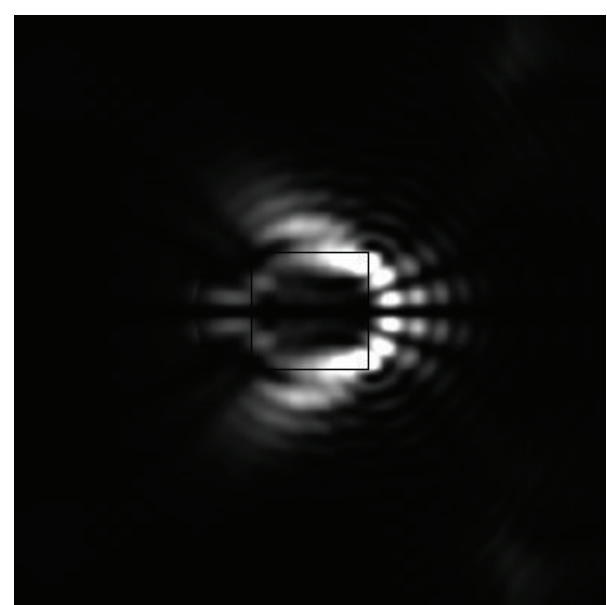

(b)

FIGURE 3: Square reconstruction using (a) proposed method and (b) conventional method [19]. Black line is the original square. 

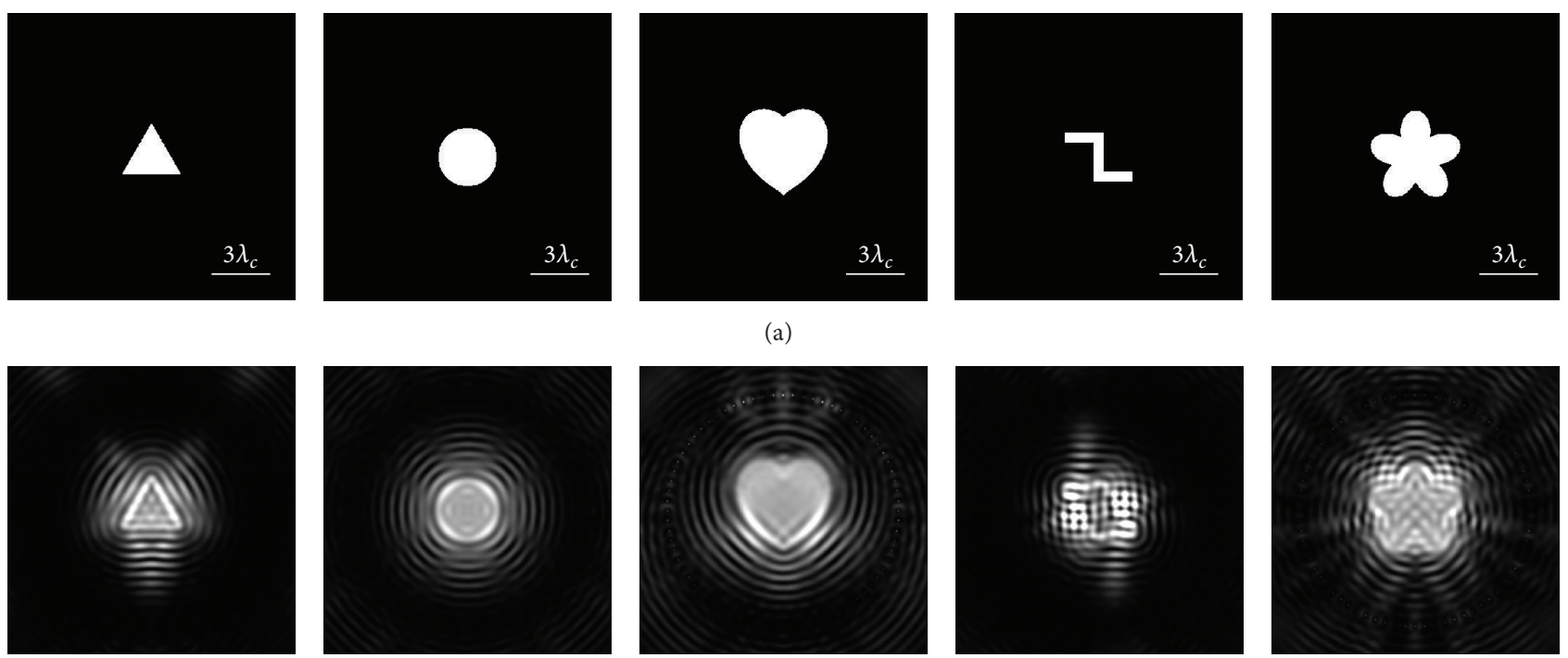

(a)
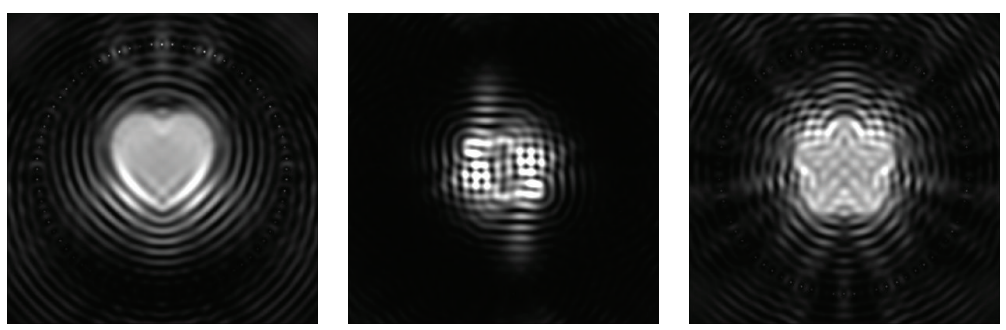

(b)

FIGURE 4: Reconstruction of some basic geometries. (a) and (b) depict original shapes (scale bar is $3 \lambda_{c}$ ) and reconstructed images. From left to right, the triangle, cylinder, heart, z-like, and flower shapes, respectively, are depicted.

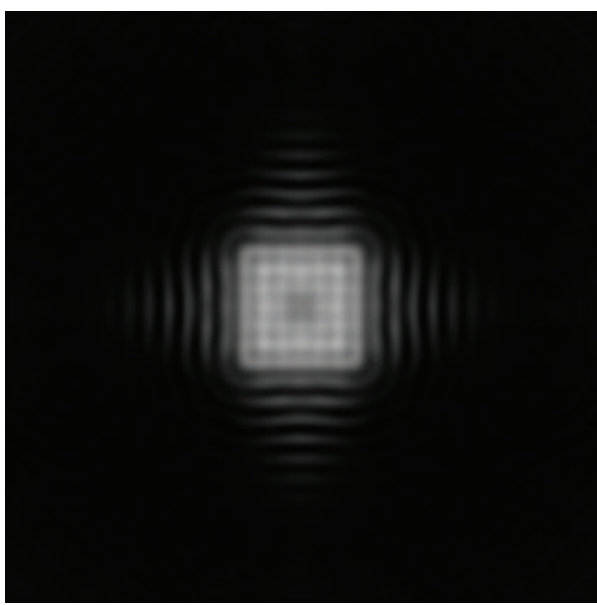

(a)

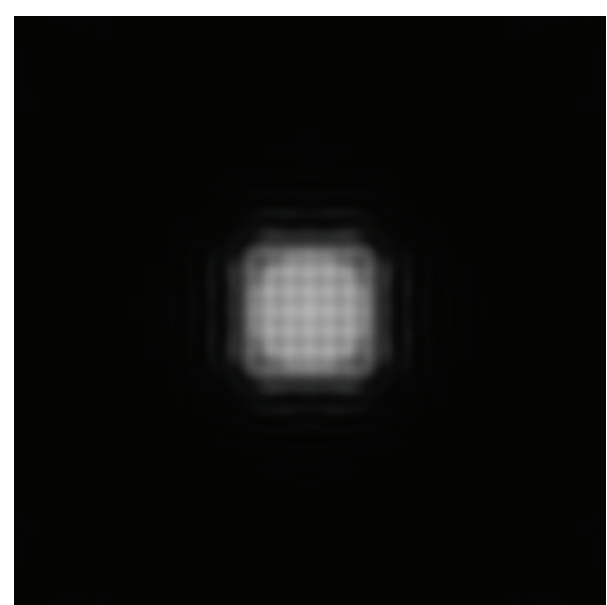

(b)

FIGURE 5: Reconstruction of a square of refractive indices (a) 2 and (b) 1.5 . The side of the square is $3 \lambda_{c}$.

are clearly reconstructed. Using additional pulses in other directions would further improve the imaging quality, but would require more measurements in the observation step.

To compare our imaging quality with the conventional interference technique [19], in Figure 3, we plot the square reconstruction with the original shape line using (a) our method and (b) the conventional method. As shown in Figure 3, our result accurately captures the size and boundary, whereas the conventional method merely reconstructs the rough shape. Thus the crucial difference between the proposed method and the conventional approach is the reproducibility of the detailed geometry.

2.4. Numerical Verifications. We verify the imaging qualities of our interference technique with respect to changes of the object shape, refractive index, and incident polarization. First we verify our approach for other basic geometries. Figure 4 shows (a) original shapes and (b) reconstructed images for triangle, cylinder, heart, $\mathrm{z}$-like, and flower shapes. The refractive indexes inside and outside the object are 3.5 and 1, respectively. Successful images can be obtained not only for the straight-edged shape (triangle), but also for the curvilinear objects (cylinder and heart). Although the $\mathrm{z}$ like shape is not axisymmetric, its geometry is successfully reconstructed (the small distortion of the shape is caused by the multiple scattering discussed in Section 3.2). In contrast, the dips in the flower image are comparatively obscure. This is because the incident pulses cannot effectively arrive in the dips smaller than $\lambda_{c}$. Details of imaging such as small features are discussed in Section 3.1. 


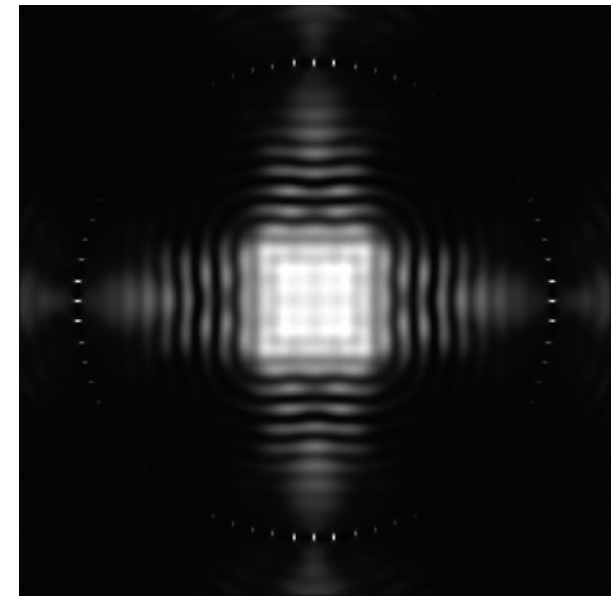

(a)

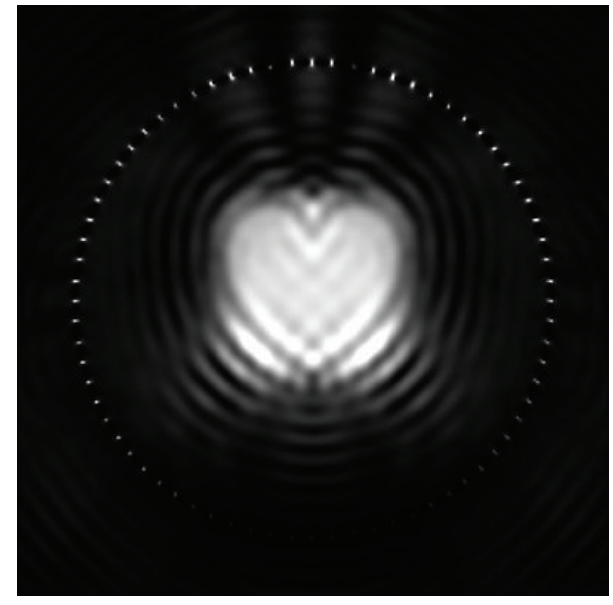

(b)

FIGURE 6: Reconstruction of (a) square and (b) heart shape in the TM mode (original shapes are given in Figures 2 and 4).

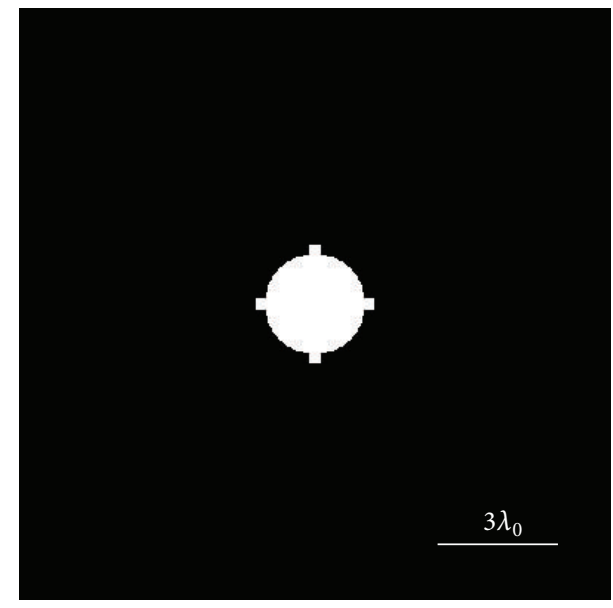

(a)

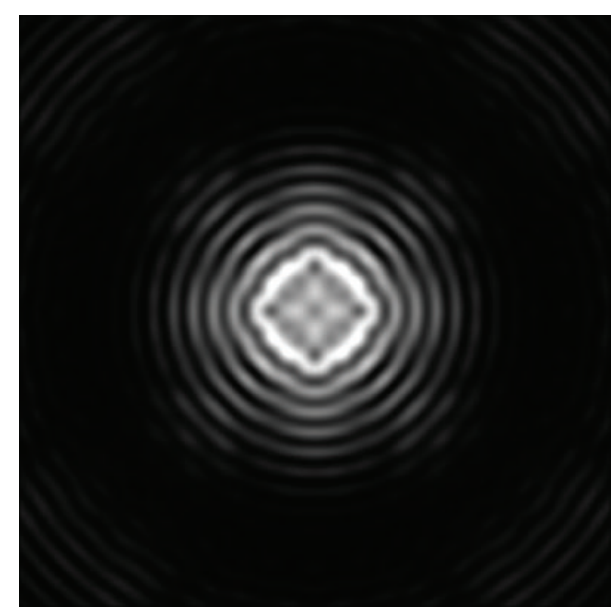

(c)

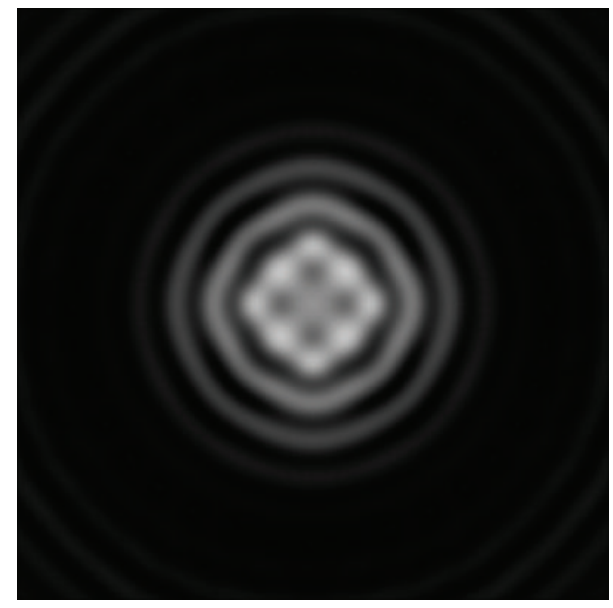

(b)

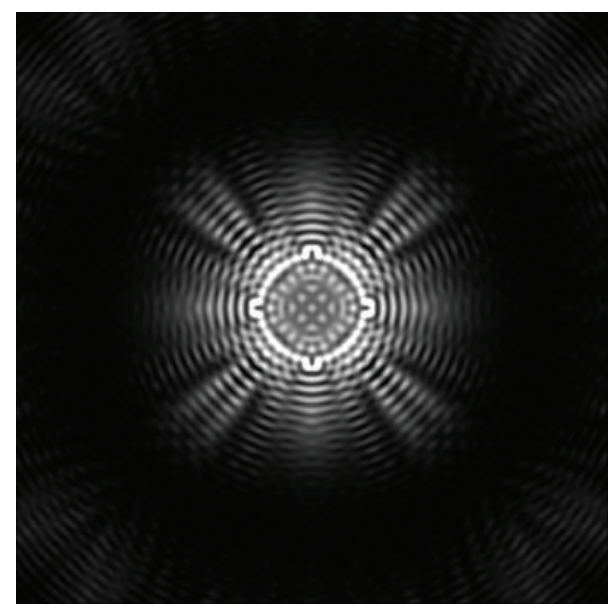

(d)

FIgURE 7: Reconstruction of a square with small bumps (width is $\lambda_{0} / 4$ ). (a) Original object (scale bar is $3 \lambda_{0}$ ). (b), (c), and (d) depict the reconstructed images for different center wavelengths, $\lambda_{c}=2 \lambda_{0}, \lambda_{0}$, and $\lambda_{0} / 2$, respectively. 


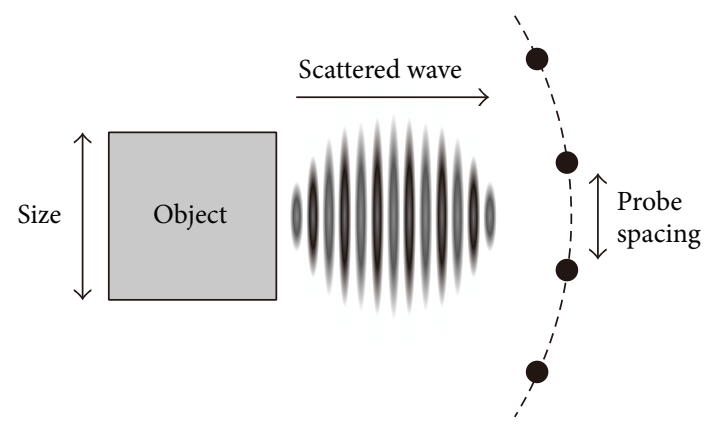

FIGURE 8: Scattering by an object larger than the incident wavelength. The object size and the measurement probe spacing are indicated.

We test our approach for other refractive indices. Figure 5 shows the imaging results for a square of refractive index (a) 2 (silicon nitride in microwave region) and (b) 1.5 (silicon dioxide in microwave region). The side of the square is $3 \lambda_{c}$. The original geometry is correctly detected for both refractive indices. Because the equiphase plane in interference patterns is formed by the object shape, the geometry can be reconstructed unless the refractive index is too low to make a detectable reflection.

The proposed approach can be easily applied to the transverse magnetic (TM) mode, in which the electromagnetic fields reduce to $H_{z}, E_{x}$, and $E_{y}$. The $E_{z}$ fields propagating along the $x$ - and $y$-axes in the TE mode are replaced by the $E_{x}$ and $E_{y}$ fields, respectively, in the TM mode. In the observation step, both the scattered $E_{x}$ and $E_{y}$ fields are detected on the measurement points. In the imaging step, we visualize the $H_{z}$ intensity after backwards propagation, calculated from the input $E_{x}$ and $E_{y}$ fields at the observation points. Figure 6 shows the scattered $H_{z}$ intensity distribution for (a) square and (b) heart shape reconstructions in the TM mode (original shapes are given in Figures 2 and 4). Since both $E_{x}$ and $E_{y}$ fields are sampled on the probes, the reconstructions of the square (with boundaries parallel to the axes) and heart shape (with boundaries that are not parallel to the axes) are not inferior to the TE one (see Figure 4).

\section{Sensitivity to Small and Multiple Objects}

In this section, we examine the sensitivity of the proposed multipulse interference technique to small and multiple objects. We show that the minimum size of detectable objects is limited by the incident wavelength and the spacing of the measurement points. Adaptability for practical problems is tested by trying to detect multiple objects.

3.1. Minimum Size of Detectable Objects. In principle, the minimum size of objects detected by electromagnetic imaging is restricted by the incident wavelength, because information about subwavelength features is carried by evanescent waves [22]. But since we use a Gaussian pulse which has a wideband spectrum, features smaller than the center wavelength are detectable. In Figure 7, we show the imaging results for a cylinder with small bumps using different center wavelengths, $\lambda_{c}=2 \lambda_{0}, \lambda_{0}$, and $\lambda_{0} / 2\left(\lambda_{0}\right.$ is a constant). The width of bumps is $\lambda_{0} / 4$. The refractive indices inside and outside the object are 3.5 and 1 , respectively. Figure 7 (a) depicts the original object (scale bar is $3 \lambda_{0}$ ). Figures $7(b)$, $7(\mathrm{c})$, and $7(\mathrm{~d})$ show the results for $\lambda_{c}=2 \lambda_{0}, \lambda_{0}$, and $\lambda_{0} / 2$, respectively. When the short wavelength (shorter than the bump size) components are very small in the incident pulse, the bumps cannot be accurately reconstructed as shown in Figure 7(b). As the incident wavelength is decreased, as shown in Figures 7(c) and 7(d), the bumps become clearly detectable.

When the object is much larger than the incident wavelength, the minimum size of detectable objects is constrained by the spacing of the measurement probe array. As shown in Figure 8, a large object produces a scattered wave of similar width to its size without the strong diffraction. In order to capture the scattered wave passing through the gap between measurement points, the spacing must be no greater than the object size. We show the imaging results for a square using different probe spacing in Figure 9. Figure 9(a) depicts the original square of side $s=4 \lambda_{c}$ and of refractive index 3.5. Figures 9(b), 9(c), and 9(d) show the reconstructed images for the probe spacing of $s, 1.5 \mathrm{~s}$, and $2 \mathrm{~s}$, respectively. Using a spacing of $s$, we successfully obtain the original shape as shown in Figure 9(b). Using a spacing of $1.5 \mathrm{~s}$, the imaging quality is greatly degraded as shown in Figure 9(c). In Figure 9(d) with a spacing of $2 \mathrm{~s}$, we can no longer discriminate the boundaries parallel to the $x$-axis because a large part of scattered field propagating along $y$-axis misses the probe array.

3.2. Multiple Objects. We verify our approach for detecting multiple objects. Figure 10 shows the imaging for multiple objects. In Figure 10(a), we depict two cylinders of radius $1.5 \lambda_{c}$ and of refractive index 3.5. Figure 10(b) shows the imaging result for these two cylinders. We can discern the two cylinders, but the area between the objects is comparatively unclear. This is because the primary waves are multiply scattered. When the wave scattered by a cylinder propagates along $y$-axis, it impinges on the other cylinder. The longer distance between objects has the smaller effect with each other in reconstruction, but basically in the inverse calculation such multiple scatterings cannot be captured without information about the original object distributions.

In general, however, the objects to be detected are buried with noisy structures which induce multiple scattering. In order to simulate such conditions, we configure a square surrounded by randomly arrayed particles in Figure 10(c). The side and refractive index of the square are $3 \lambda_{c}$ and 3.5 , respectively. The radius and refractive index of the particles are $\lambda_{c} / 10$ and 1.5 , respectively. The total area of 30 particles is $10 \%$ of the square. Figure $10(\mathrm{~d})$ shows the imaging result for the square surrounded by noise particles. Although the centrally placed square is detectable, multiple scatterings by noise particles degrade the reconstruction compared with the result for the same square in free space (Figure 2). Especially in upper right corner of the square, the closely 


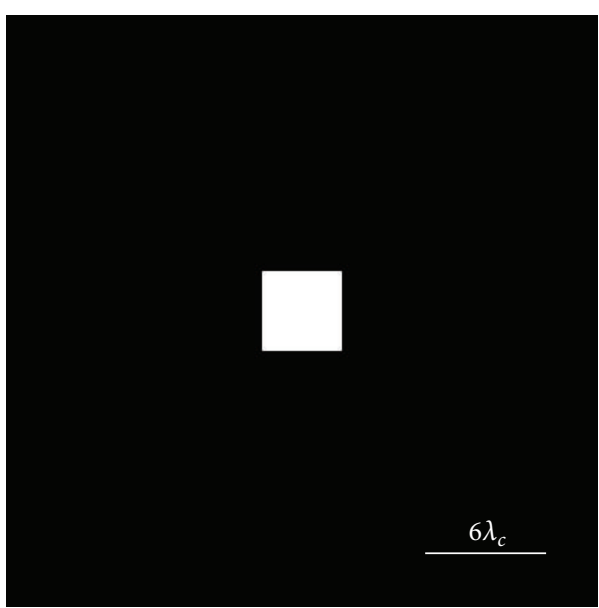

(a)

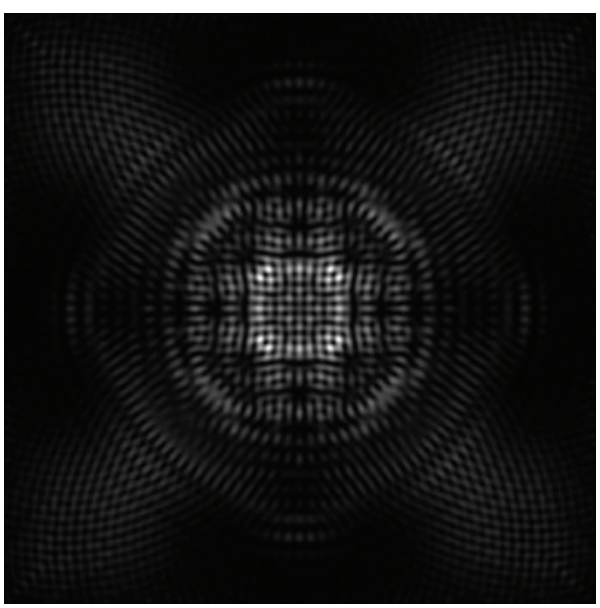

(c)

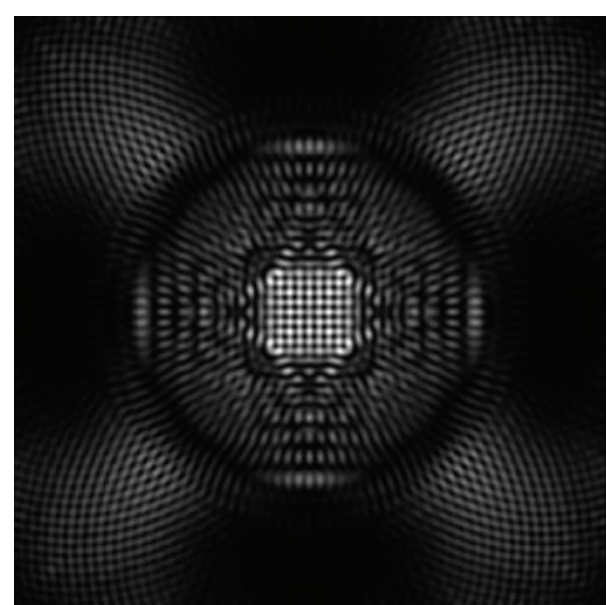

(b)

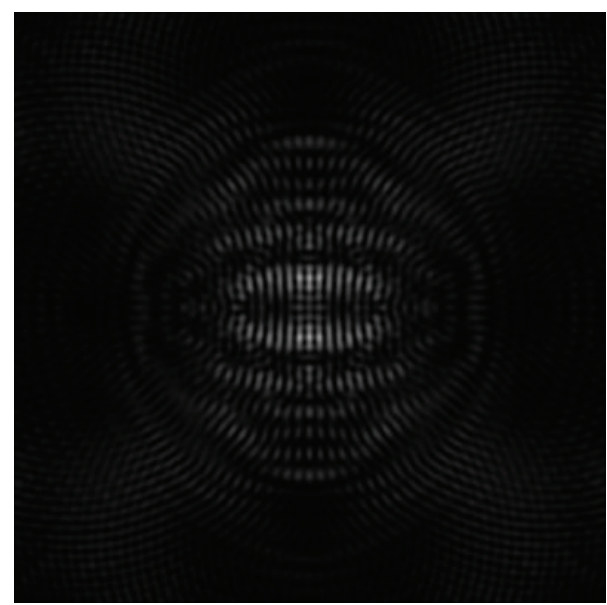

(d)

Figure 9: Reconstruction of a square using different probe spacing. (a) Original square of side $s=4 \lambda_{c}$ (scale bar is $6 \lambda_{c}$ ). (b), (c), and (d) depict the reconstructed images for probe spacing of $s, 1.5 \mathrm{~s}$, and $2 \mathrm{~s}$, respectively.

spaced particles degrade the imaging. The reconstructions for a square with fewer and more surrounding particles are shown in Appendix.

This result for noisy particles suggests that the multipulse interference technique is not suitable for nested structures. Figure 10(e) depicts a cylinder surrounded by a square (both of refractive indices are 3.5). The radius of the cylinder is $\lambda_{c}$. The side and thickness of the square are $2 \lambda_{c}$ and $\lambda_{c} / 2$, respectively. In such a nested structure, only the outmost geometry is detectable because the scattered fields due to the inner structures are trapped by the outer structures. As shown in Figure 10(f), the inner structure cannot be detected, but the outmost square is clearly detected.

\section{Conclusion}

A multipulse interference technique for two-dimensional geometries has been proposed based on the inverse FDTD method. The proposed approach, using the sum of separately calculated intensity distributions, improves the imaging quality compared with the conventional method. The interference technique is based on the principle that the equiphase plane caused by reflection delay is parallel to the object surface and shows the robustness for changes of the object shape, refractive index, and incident polarization. The minimum size of detectable objects is similar in size to the incident wavelength due to the diffraction limit and is larger than the probe spacing needed to capture a scattered wave that is not greater than the object size. Thus, the shorter the wavelength and the closer the probe spacing, the better the imaging quality in our method. Multiple objects are detectable unless the multiple scattering is dominant. Noise particles that account up to about $10 \%$ of the object area within one object width of its surface are acceptable in our reconstruction. For nested structures, only the outmost geometry is detectable because the scattered fields due to the inner structures are trapped by the outer structures.

In future work we will try to extend the proposed method into the three-dimensional problems and the pseudo-spectral 


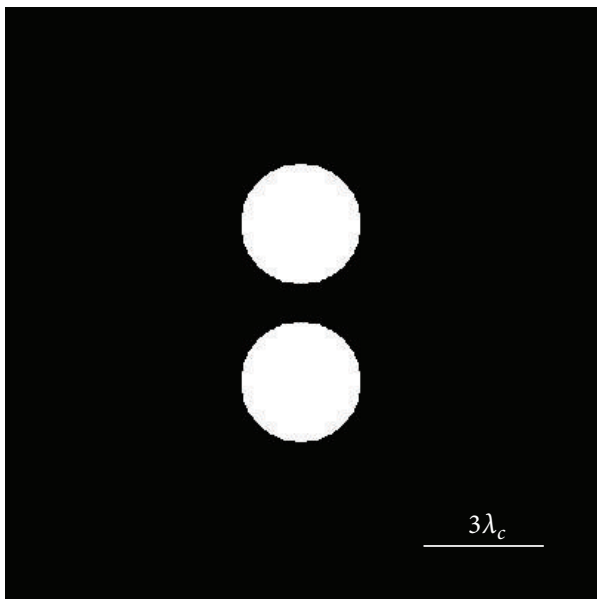

(a)

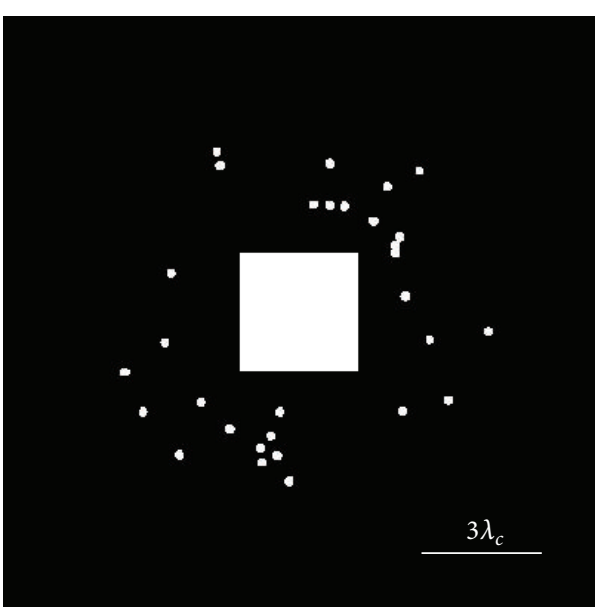

(c)

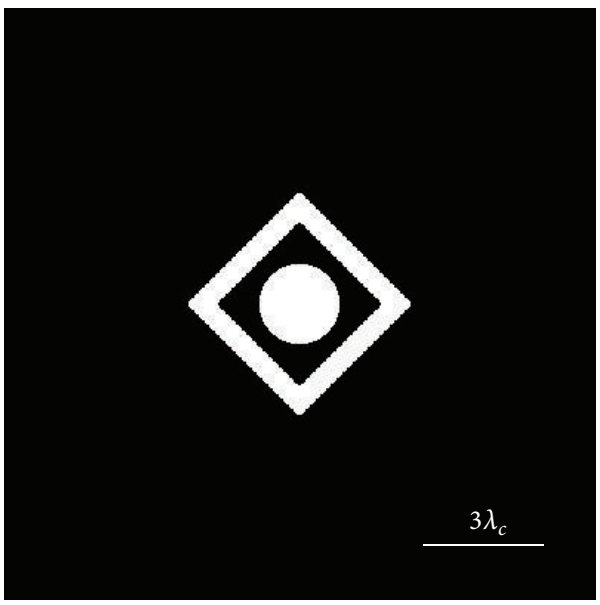

(e)

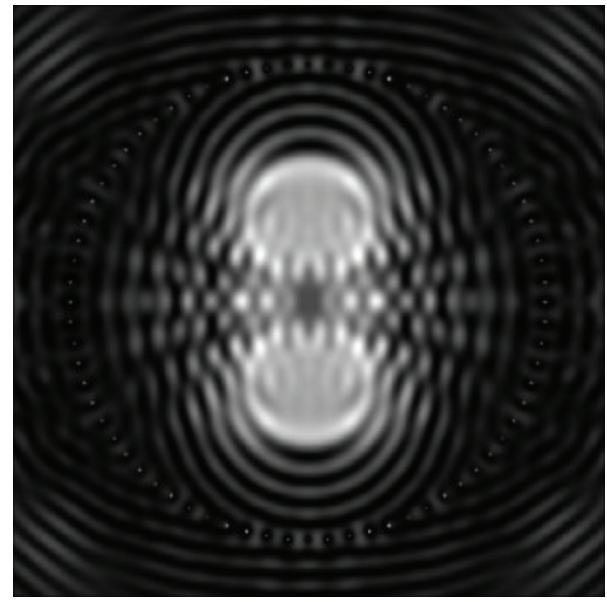

(b)

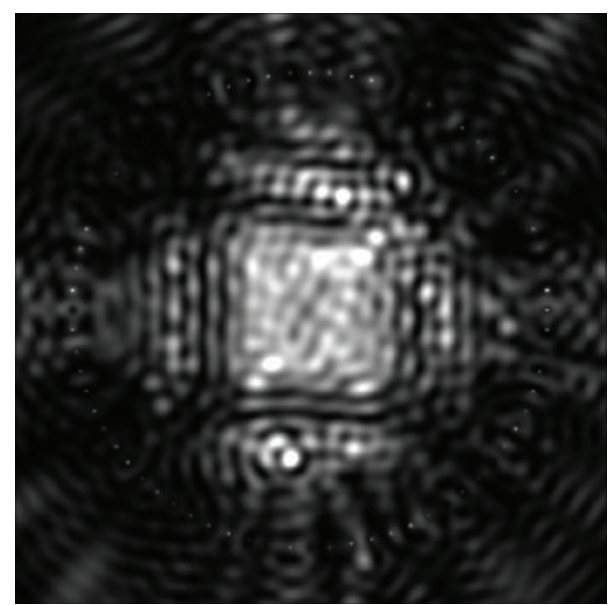

(d)

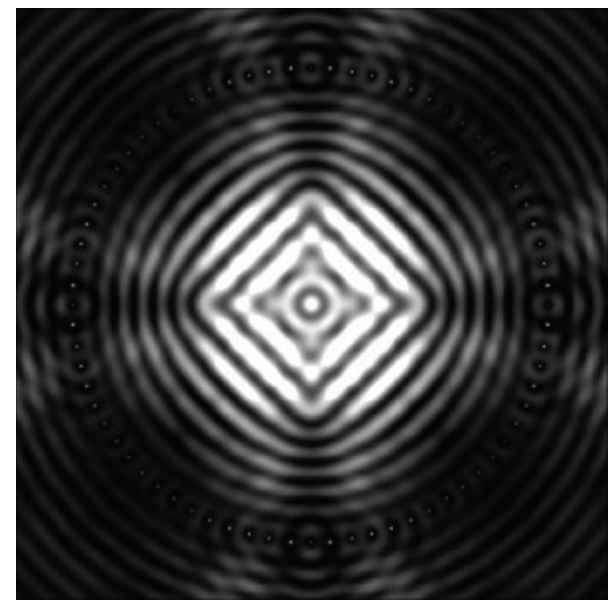

(f)

FIGURE 10: Reconstruction of multiple objects. (a) and (b) are original and reconstructed shapes of two cylinders, respectively. (c) and (d) are original and reconstructed shapes of a square surrounded by randomly arrayed particles, respectively. (e) and (f) are original and reconstructed shapes of a cylinder surrounded by a square. Scale bars are $3 \lambda_{c}$. 
$5 \%$
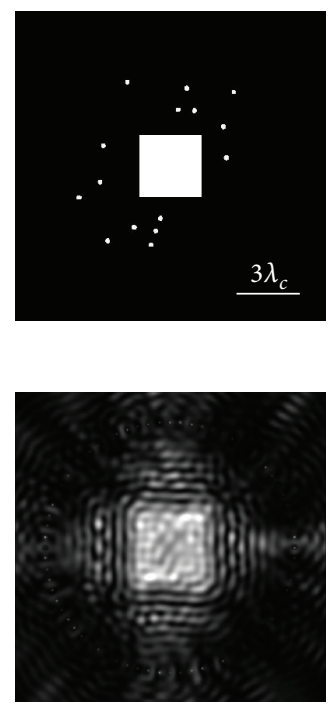

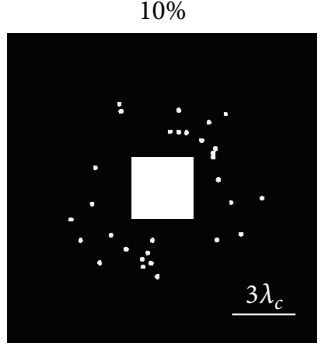

$15 \%$

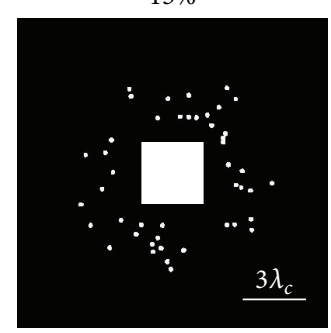

(a)
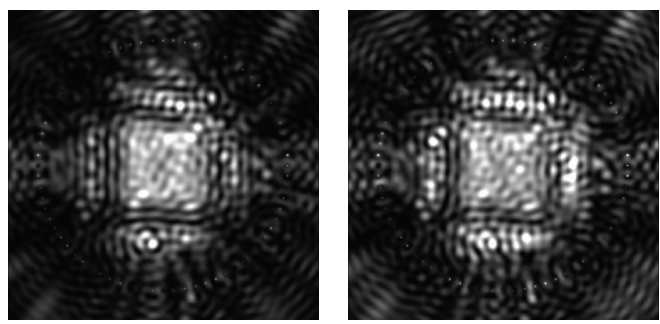

$20 \%$
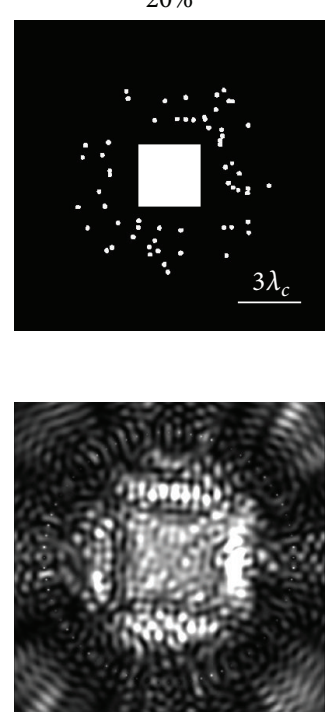

$30 \%$
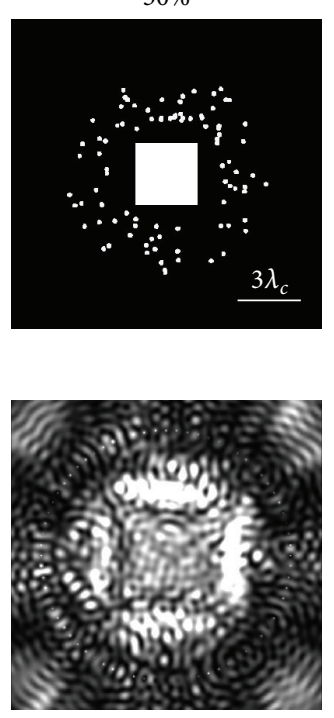

(b)

FIGURE 11: Reconstruction for a square with the different number of surrounding particles. (a) and (b) depict original shapes (scale bar is $3 \lambda_{c}$ ) and reconstructed images. From left to right, the total area of particles is $5 \%, 10 \%, 15 \%, 20 \%$, and $30 \%$ of the square, respectively. Scale bars are $3 \lambda_{c}$.

time-domain method $[23,24]$ that can improve the computational cost.

\section{Appendix}

We examine the imaging quality for the different number of surrounding particles. Figure 11 shows (a) original objects and (b) reconstructions for a square with randomly arrayed particles when the total area of particles is $5 \%, 10 \%, 15 \%$, $20 \%$, and $30 \%$ of the square, respectively. The computational condition is the same as Section 3.2. As shown in Figure 11, the fewer the surrounding particles are, the better the shape is reconstructed. Thus the worst case to detect inner structures is the nested structure.

\section{Conflict of Interests}

The authors declare that there is no conflict of interests regarding the publication of this paper.

\section{Acknowledgments}

The authors thank the Japan Society for the Promotion of Science Fellowship Program (Grant no. 23.285) for financial support. The authors thank the Japanese Ministry of Education, Culture, Sports, Science, and Technology for financial support via the A-Step and KAKENHI grants.

\section{References}

[1] J. C. Bolomey, A. Izadnegahdar, L. Jofre, Ch. Pichot, G. Peronnet, and M. Solaimani, "Microwave diffraction tomography for biomedical applications," IEEE Transactions on Microwave Theory and Techniques, vol. 30, no. 11, pp. 1998-2000, 1982.

[2] S. R. H. Hoole, S. Subramaniam, R. Saldanha, J.-L. Coulomb, and J.-C. Sabonnadiere, "Inverse problem methodology and finite elements in the identification of cracks, sources, materials, and their geometry in inaccessible locations," IEEE Transactions on Magnetics, vol. 27, no. 3, pp. 3433-3443, 1991.

[3] P. M. Meaney, K. D. Paulsen, and J. T. Chang, "Near-field microwave imaging of biologically-based materials using a monopole transceiver system," IEEE Transactions on Microwave Theory and Techniques, vol. 46, no. 1, pp. 31-45, 1998.

[4] K. M. Golden, D. Borup, M. Cheney et al., "Inverse electromagnetic scattering models for sea ice," IEEE Transactions on Geoscience and Remote Sensing, vol. 36, no. 5, pp. 1675-1704, 1998.

[5] A. Qing, C. K. Lee, and L. Jen, "Electromagnetic inverse scattering of two-dimensional perfectly conducting objects by real-coded genetic algorithm," IEEE Transactions on Geoscience and Remote Sensing, vol. 39, no. 3, pp. 665-676, 2001.

[6] Y.-S. Chung, C. Cheon, and S.-Y. Hahn, "Reconstruction of dielectric cylinders using FDTD and topology optimization technique," IEEE Transactions on Magnetics, vol. 36, no. 4, pp. 956-959, 2000.

[7] I. T. Rekanos, "Inverse scattering in the time domain: an iterative method using an FDTD sensitivity analysis scheme," IEEE Transactions on Magnetics, vol. 38, no. 2, pp. 1117-1120, 2002.

[8] X. Chen, D. Liang, and K. Huang, "Microwave imaging 3$\mathrm{D}$ buried objects using parallel genetic algorithm combined with FDTD technique," Journal of Electromagnetic Waves and Applications, vol. 20, no. 13, pp. 1761-1774, 2006.

[9] M. Donelli, D. Franceschini, P. Rocca, and A. Massa, “Threedimensional microwave imaging problems solved through an 
efficient multiscaling particle swarm optimization," IEEE Transactions on Geoscience and Remote Sensing, vol. 47, no. 5, pp. 1467-1481, 2009.

[10] K. S. Yee, "Numerical solution of initial boundary value problems involving Maxwell's equations in isotropic media," IEEE Transactions on Antennas \& Propagation, vol. 14, no. 3, pp. 302307, 1966.

[11] A. Taflove and S. C. Hagness, Computational Electrodynamics: The Finite-Difference Time-Domain Method, Artech House, 3rd edition, 2005.

[12] K. Belkebir, R. E. Kleinman, and C. Pichot, "Microwave imaging-location and shape reconstruction from multifrequency scattering data," IEEE Transactions on Microwave Theory and Techniques, vol. 45, no. 4, pp. 469-476, 1997.

[13] C.-C. Chiu and W.-T. Chen, "Electromagnetic imaging for an imperfectly conducting cylinder by the genetic algorithm," IEEE Transactions on Microwave Theory and Techniques, vol. 48, no. 11, pp. 1901-1905, 2000.

[14] S. Caorsi, A. Massa, and M. Pastorino, "A computational technique based on a real-coded genetic algorithm for microwave imaging purposes," IEEE Transactions on Geoscience and Remote Sensing, vol. 38, no. 4, pp. 1697-1708, 2000.

[15] S. Caorsi and M. Pastorino, "Two-dimensional microwave imaging approach based on a genetic algorithm," IEEE Transactions on Antennas and Propagation, vol. 48, no. 3, pp. 370-373, 2000.

[16] S. Caorsi, A. Costa, and M. Pastorino, "Microwave imaging within the second-order born approximation: stochastic optimization by a genetic algorithm," IEEE Transactions on Antennas and Propagation, vol. 49, no. 1, pp. 22-31, 2001.

[17] M. Pastorino, A. Massa, and S. Caorsi, "A microwave inverse scattering technique for image reconstruction based on a genetic algorithm," IEEE Transactions on Instrumentation and Measurement, vol. 49, no. 3, pp. 573-578, 2000.

[18] E. Abenius and B. Strand, "Solving inverse electromagnetic problems using FDTD and gradient-based minimization," International Journal for Numerical Methods in Engineering, vol. 68, no. 6, pp. 650-673, 2006.

[19] K. Chakrabarti and J. B. Cole, "Simulation study on the detection of size, shape and position of three different scatterers using Non-standard time domain time inverse Maxwell's algorithm," Optics Express, vol. 18, no. 5, pp. 4148-4157, 2010.

[20] A. Taflove and M. E. Brodwin, "Numerical solution of steady-state electromagnetic scattering problems using the time-dependent Maxwell's equations," IEEE Transactions on Microwave Theory and Techniques, vol. 23, no. 8, pp. 623-630, 1975.

[21] J. B. Cole, "The statistical mechanics of image recovery and pattern recognition," American Journal of Physics, vol. 59, no. 9, pp. 839-842, 1991.

[22] J. B. Pendry, "Negative refraction makes a perfect lens," Physical Review Letters, vol. 85, no. 18, pp. 3966-3969, 2000.

[23] Q. H. Liu, "The PSTD algorithm: a time-domain method requiring only two cells per wavelength," Microwave and Optical Technology Letters, vol. 15, no. 3, pp. 158-165, 1997.

[24] C. Liu, R. L. Panetta, and P. Yang, "Application of the pseudospectral time domain method to compute particle singlescattering properties for size parameters up to 200," Journal of Quantitative Spectroscopy and Radiative Transfer, vol. 113, no. 13, pp. 1728-1740, 2012. 

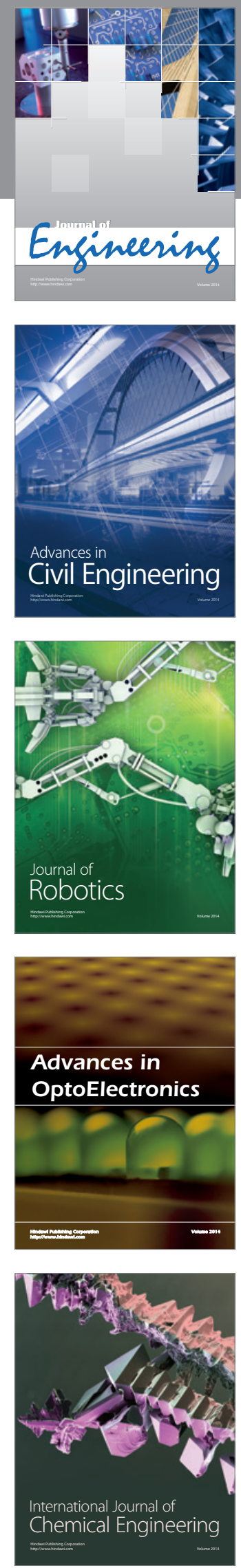

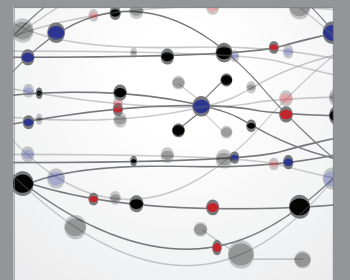

The Scientific World Journal
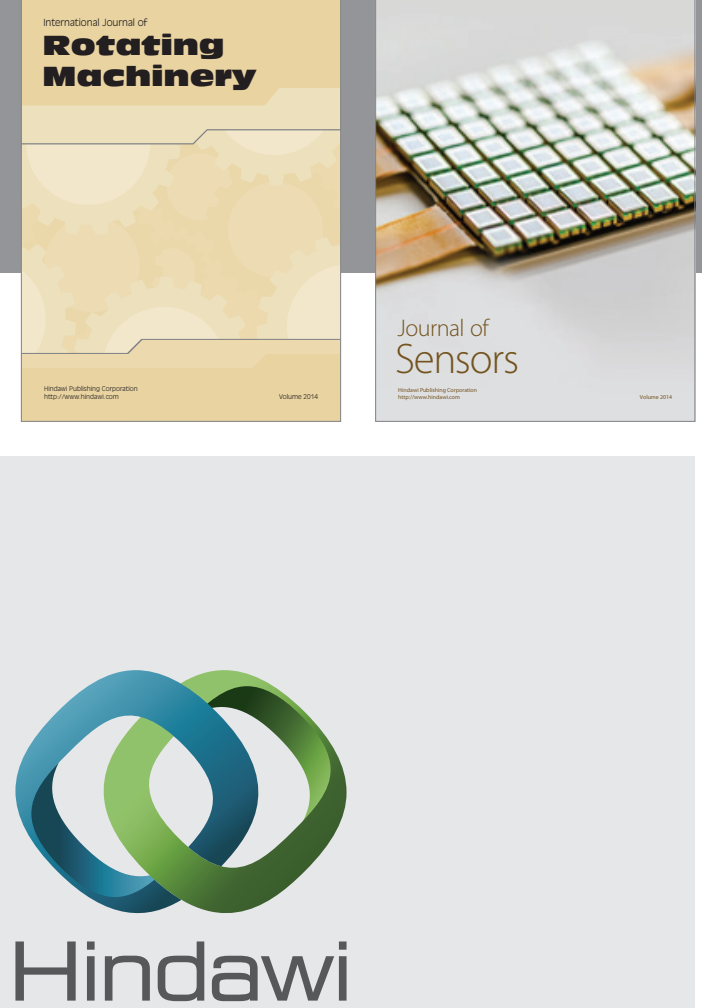

Submit your manuscripts at http://www.hindawi.com
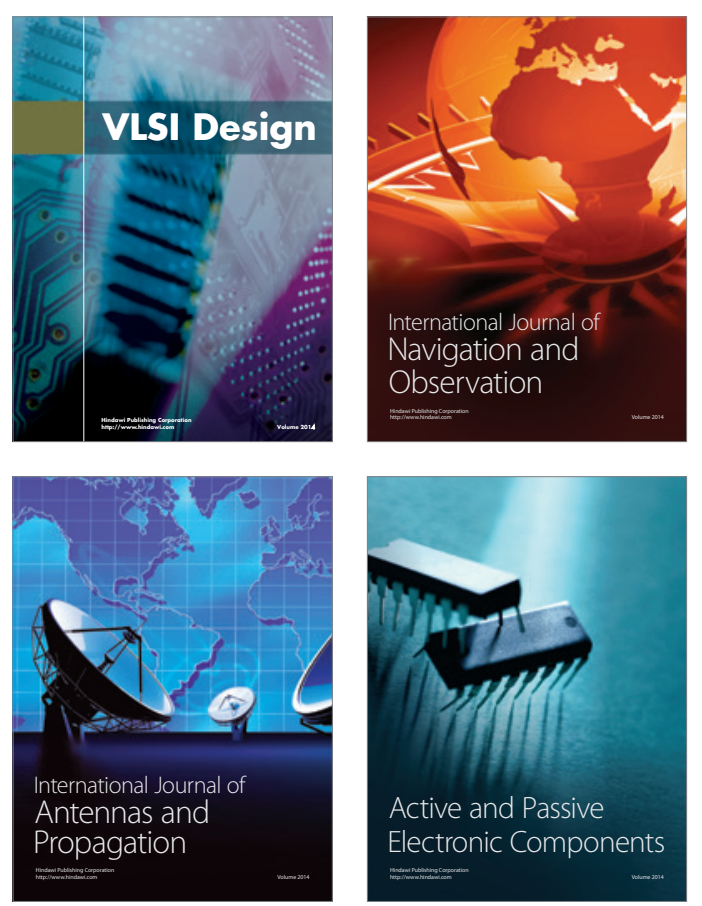
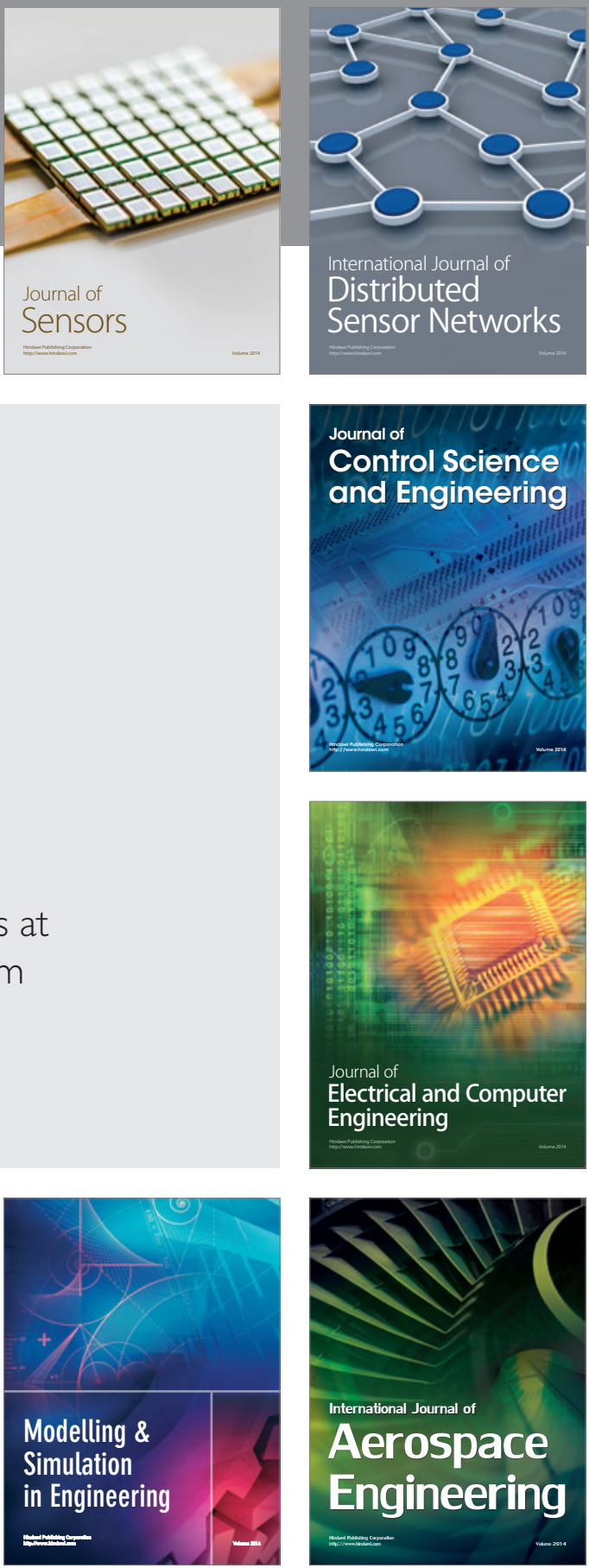

Journal of

Control Science

and Engineering
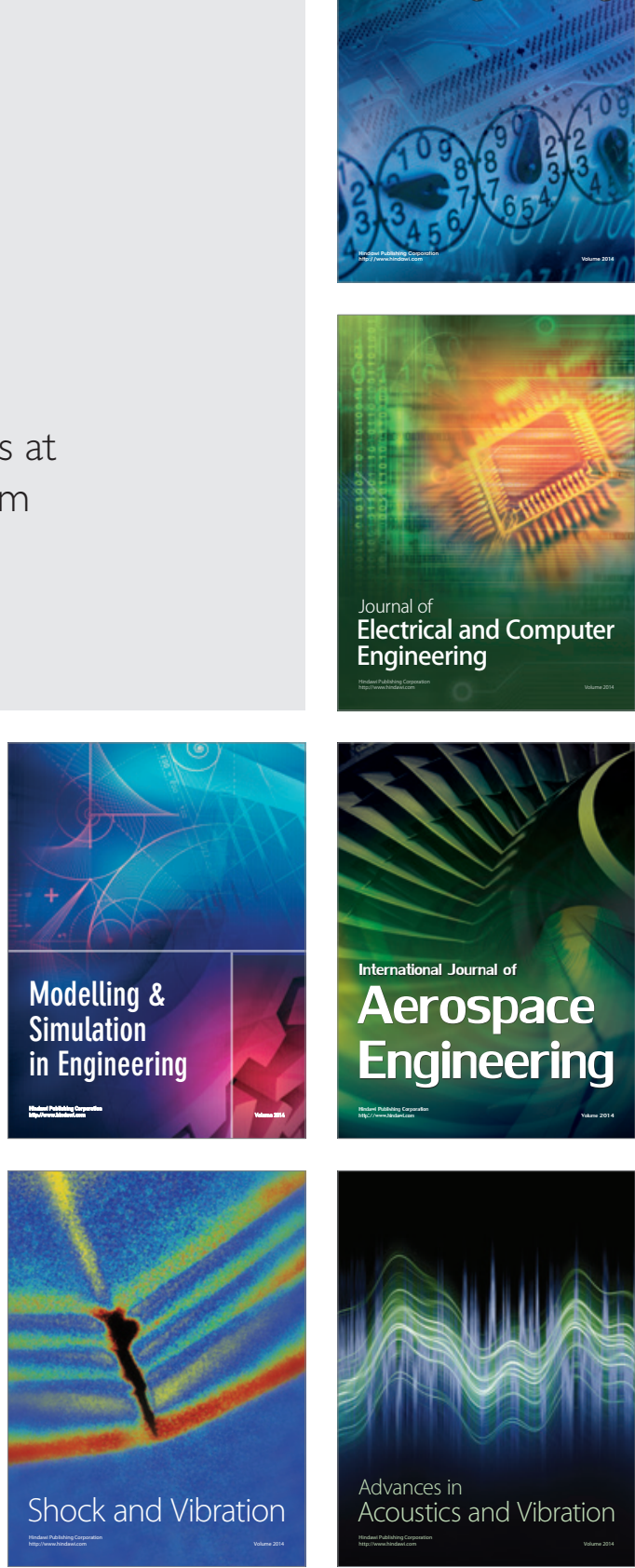